\title{
Wheel slip ratio regulation for investigating the vehicle's dynamic behavior during braking and steering input
}

\author{
Ali Shahabi, Amir Hossein Kazemian*, Said Farahat, and Faramarz Sarhaddi \\ Department of Mechanical Engineering, University of Sistan and Baluchestan, Zahedan, Iran
}

Received: 10 August 2020 / Accepted: 14 February 2021

\begin{abstract}
In this study, the vehicle's dynamic behavior during braking and steering input is investigated by considering the quarter-car model. The case study for this research is a Sport-Utility Vehicle (SUV) with the anti-lock braking system (ABS) and nonlinear dynamic equations are considered for it along with Pacejka tire model. Regulating the wheel slip ratio in the optimal value for different conditions of the road surface (dry, wet and icy) during braking is considered as the ABS control strategy. In order to regulating the wheel slip ratio in the optimal value, an intelligent adaptive fuzzy controller that can perform online parameter estimation is considered. In this regard, the proposed controller tracks the optimal wheel slip ratio with changing the condition of the road surface from dry to wet and icy. The adaptive fuzzy controller consists of linguistic base, inference engine and defuzzifier section. The wheel slip ratio and vehicle longitudinal acceleration are selected as inputs of the controller, controller adapter and detector of the road surface condition. During braking and steering input, effective parameters of the wheel that are affected on the vehicle's dynamic behavior and its stability are investigated.
\end{abstract}

Keywords: Anti-lock brake system / sport-utility vehicle / adaptive fuzzy controller / stability / online parameter estimation

\section{Introduction}

Vehicle safety is an important factor for designers, so vehicles have to meet strict safety standards in order to be allowed to enter the market. Vehicle stability is one of the most important issues in pre-crash safety. Because of higher customer demand for SUVs in recent years, many automobile companies are interested in the production of these types of automobiles. Due to high height of the center of gravity, SUVs have higher probability of instability than the other vehicles [1]. The influence of center of mass longitudinal position, tire cornering stiffness and front/rear roll stiffness ratio on the vehicle stability analyzed by Farroni et al. [2]. Hisaoka et al. [3] discussed an analysis of driver-vehicle behavior during braking in turns. The behavior of the vehicle on the lateral stable and unstable areas for calculating the probability of wheel lock during braking presented by Lenasi et al. [4]. $\mathrm{Qu}$ and Liu [5] studied on the nonlinear influences of tires on the steady-state steering behavior, dynamic response and handling stability of the vehicle. Olson et al. [6]

\footnotetext{
* e-mail: kazemian@eng.usb.ac.ir
}

considered the longitudinal braking dynamics of a twowheel vehicle model on an incline using techniques from nonlinear dynamics. Ahmadian [7] improved handling, stability and ride comfort of the vehicle by theoretical and experimental methods. Kazemian et al. [8,9] proposed the dynamic of SUVs by a new suspension system and they improved the instability of the vehicle system by proposed suspension systems. Joa et al. [10] estimated the tire slip angles under various road conditions without the prior knowledge of tire and road condition only by using onboard vehicle sensors. Cheng et al. [11] proposed an adaptive-sliding-mode observer to estimate the lateral tire force. Reiter and Wagner [12] studied on the variance of longitudinal and lateral forces and plus aligning torque of tires. Zhang et al. [13] proposed a reconfigurable control scheme for articulated stabilization of vehicles by leveraging optimization-based control techniques. Moreover, basic researches of dynamic modeling have been placed in [14-20].

In different road conditions, especially slippery roads, proper operation of the braking system can guarantee the stability of the vehicle during braking. The anti-lock braking system improves the stability and safety of the vehicle by regulating the optimal wheel slip ratio. 
Intelligent control could be useful for regulation of the wheel slip ratio to obtain ABS purposes. Lee and Zak [21] designed a genetic neural fuzzy anti-lock-brake-system controller for different types of braking maneuvers. Change of membership function characteristics in the controller to improve the fuzzy control of antiskid braking systems studied by Layne et al. [22]. Madau et al. [23] described the preliminary research and implementation of a fuzzy logic controller to control the wheel slip ratio for an anti-lock brake system. A friction monitoring system with using nonlinear and linear mathematical models for describing the friction coefficient characteristics and computing the dynamic wheel loads and longitudinal tire forces developed by Germann et al. [24]. Mirzaei et al. [25] proposed an optimized fuzzy controller for anti-lock braking systems and they considered the wheel speed and the vehicle acceleration as two input controller variables. An optimization-based on non-linear control law with increased robustness designed by Mirzaeinejad and Mirzaei [26] for a two-wheel ABS. Šabanovič et al. [27] contributed to the development of the new efficient engineering solution aimed at improving vehicle dynamics control via the antilock braking system (ABS) by estimating friction coefficient using video data. To verify functionality of an intelligent open loop fuzzy-logic-based anti-lock braking system the control method for four on-board motor drive electric sport utility vehicle a hardware-in-the-loop experiment conducted by Aksjonov et al. [28]. Zhang et al. [29] presented a novel nonlinear robust wheel slip rate tracking control strategy for autonomous vehicle with actuator dynamics. Wellstead and Pettit [30] described the analysis and subsequent redesign of an ABS using a new method for studying the dynamical behavior of piecewise linear systems. An adaptive fuzzy logic control of discretetime dynamical systems proposed in $[31,32]$ and Sakai et al. [33] designed a robust discrete-time controller. A vehicle dynamics control system developed by Zheng et al. [34] for tracking desired vehicle behavior. Applications of fuzzy set theory and fuzzy adaptive control of a first-order process with a varying gain and time constant investigated in $[35,36]$. Other important controllers for anti-lock braking systems have been placed in [37-42].

According to previous researches with changing the road surface condition, during braking the controller does not have a good performance and the wheel slip ratio is regulated in a predetermined constant value for each surface condition. On the other hand, when the road surface conditions are changed, the slip ratio of wheels is not regulated in the optimal value. For the quarter-car model of this study, when the road surface condition is changed from dry to wet and icy and vice versa, for obtaining the good performance of the brake system, the intelligent ABS adaptive fuzzy controller due to the online parameter estimation is considered. Wheel slip ratio regulation in the maximum range of longitudinal friction coefficient for different conditions of the road surface is selected as the purpose of the ABS control. During braking and steering input on a slippery road, the influence of the wheel parameters is analyzed on the vehicle's dynamic behavior.

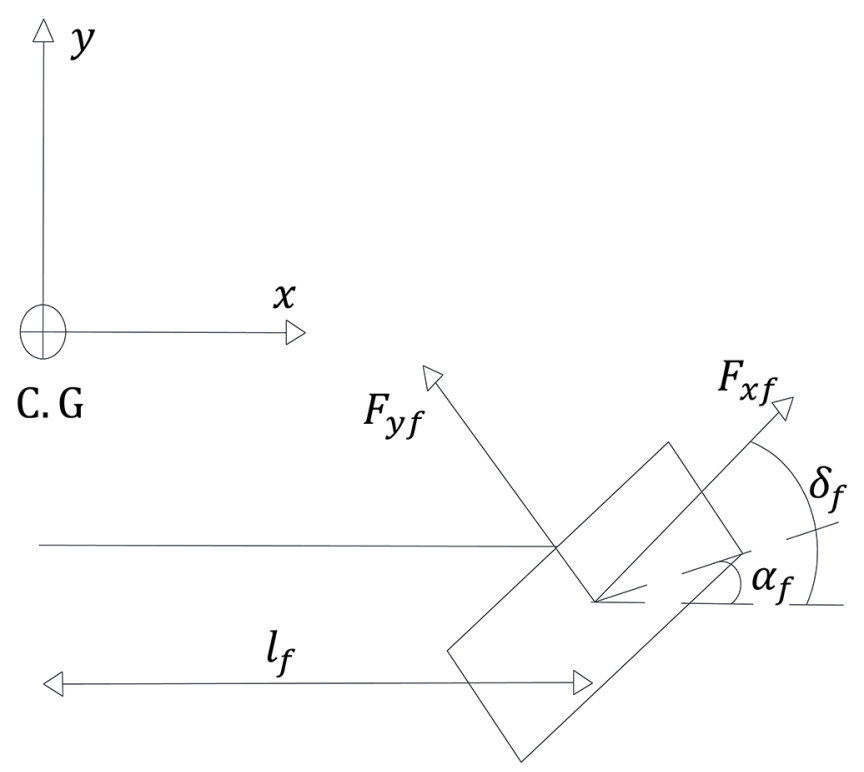

Fig. 1. Quarter-car model of the vehicle.

\section{Vehicle model and equations}

A quarter-car model is considered for investigating the dynamic analysis of the vehicle (see Fig. 1). The vehicle is passed in a path that consists of longitudinal, lateral and yaw motions by steering input. The main equations of motion for the quarter-car model can be simplified as follows [34]:

Longitudinal motion:

$\sum F_{x}=F_{x f} \cos \delta_{f}-F_{y f} \sin \delta_{f}=m\left(\dot{V}_{x}-V_{y} \dot{\varphi}\right)$

Lateral motion:

$\sum F_{y}=F_{x f} \sin \delta_{f}+F_{y f} \cos \delta_{f}=m\left(\dot{V}_{y}+V_{x} \dot{\varphi}\right)$

Yaw motion:

$$
\begin{aligned}
\sum M_{z}= & \left(F_{x f} \sin \delta_{f}+F_{y f} \cos \delta_{f}\right) l_{f} \\
& +\left(F_{x f} \cos \delta_{f}-F_{y f} \sin \delta_{f}\right) \frac{d}{2}+M_{z f}=I_{z z} \ddot{\varphi}
\end{aligned}
$$

which $m$ denotes the mass of the vehicle, $I$ is the moment of inertia, $\delta$ is the steering input and $V$ is the vehicle velocity. $\varphi$ is the yaw angle, $M_{z f}$ is self-aligning moment of the front wheel and subscript $f$ shows the front wheel. $l_{f}$ is the distance from the center of gravity to front axle and $d$ is the vehicle width.

\subsection{Lateral and longitudinal forces of the tire}

The Pacejka non-linear Magic Formula is considered to evaluate the lateral and the longitudinal forces of the tire $[19,24,34,38]$. The lateral slip angle is difference between the direction of tire longitudinal axis and the direction of 
tire velocity vector in the $x y$ plane (Fig. 2). Therefore the lateral slip angle $(\alpha)$ is considered as [19,34]:

$$
\alpha_{f}=\arctan \left[\left(V_{y}+\dot{\varphi} \cdot l_{f}\right) /\left(V_{x}+\dot{\varphi} \cdot d / 2\right)\right]-\delta_{f}
$$

The output of the Magic Formula under some inputs such as lateral slip angle and vertical force of the wheel (static load) is introduced as $[19,34]$ :

$$
Y(x)=D \sin (C \arctan (B x-E(B x-\arctan (B x))))
$$

which, $Y(x)$ is the lateral force $\left(F_{y}\right)$ with the lateral slip angle and $B, C, D$ and $E$ are the constant parameters of the Magic Formula. In this research the lateral force is considered as $F_{y f}=\left(K_{c} \alpha_{f}\right) N$ that $K_{c}$ is the sideslip force stiffness of the tire and $N$ is the tire normal load [42]. The longitudinal force $\left(F_{x}\right)$ is considered as a function of the longitudinal friction coefficient as equation (6) [24,34]:

$$
F_{x f}=-\mu(S) \cdot m g
$$

In equation (6), $\mu(S)$ related to the longitudinal friction coefficient and it is a function of the wheel slip ratio $(S)$. The formula of $\mu(S)$ is defined as equation (7) [19,24,38]:

$\mu(S)=D \sin \left(C \arctan (B(1-E)(S))+\left(\frac{E}{B} \arctan (B(S))\right)\right)$

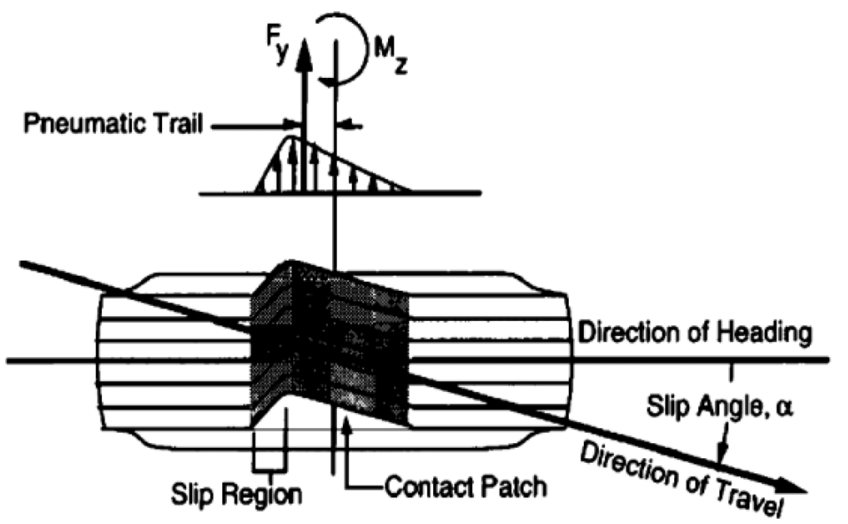

Fig. 2. Tire characteristics in contact patch area [19].
Figure 3 shows typical variations of the longitudinal and lateral friction coefficients with respect to the wheel slip ratio $(\mu(S)$ curves, Fig. 3a [23]), $\mu(S)$ curves for different conditions of the road surface (Fig. 3b [24]) and comparison of $\mu(S)$ curves for tire models (Fig. 3c [24]). Figure 3c also shows different models of the tire and it is proved that the Pacejka Formula has the most appropriate results for all range of wheel slip ratio than the other tire models such as polynomial model.

\subsection{Wheel rotational equation}

The rotational equation of the wheel is obtained as $[24,34]$ :

$$
I_{w} \dot{\omega}_{w}=-T_{b}-r_{w} F_{x}
$$

which $r_{w}$ is the radius of the wheel, $T_{b}$ is the brake torque that acts on the wheel, $\omega_{w}$ and $I_{w}$ are angular velocity and moment of inertia of the wheel; respectively. During braking, the wheel slip ratio $(S)$ is defined as [34,38]:

$$
S=\frac{r_{w} \omega_{w}-V_{x}}{V_{x}}
$$

The slip ratio shows the wheel slip according to the longitudinal velocity of the vehicle and angular velocity of the wheel and $0 \leq|S| \leq 1$. When $\omega_{w}=0$ then $|S|=1$ and the wheel lock-up will be occurred.

\subsection{Self-aligning moment of tires}

The self-aligning moment $\left(M_{z}\right)$ indicates the tendency of the tire to accept the steering input around the vertical axis. This moment is resulted from the deformation of tire in contact patch area (Fig. 2). The self-aligning moment is defined as the product between the lateral force and the pneumatic trail. The pneumatic trail is resulted from the lateral slip angle and the pneumatic trail is defined with the Magic Formula as [19,20,34]:

$$
Y(x)=D \cos (C \arctan (B x-E(B x-\arctan (B x))))
$$

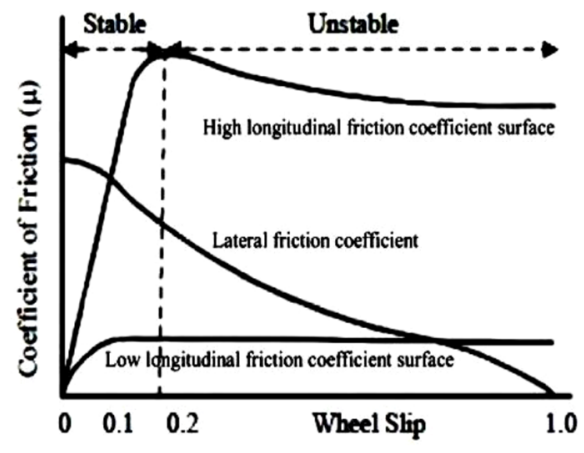

(a)

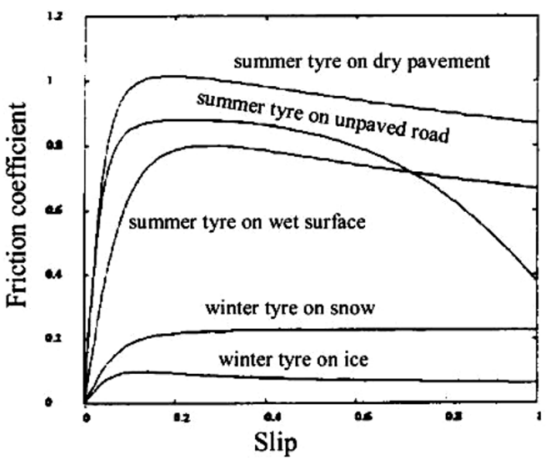

(b)

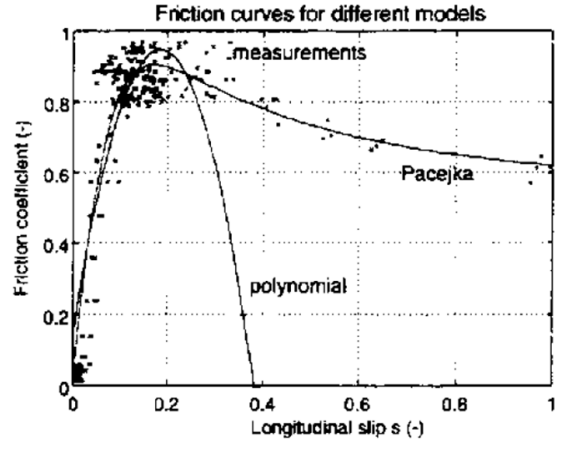

(c)

Fig. 3. $\mu(S)$ curves: (a) variations of friction coefficients with respect to the wheel slip ratio, (b) for different conditions of the road surface and (c) comparison for different models of the tire. 


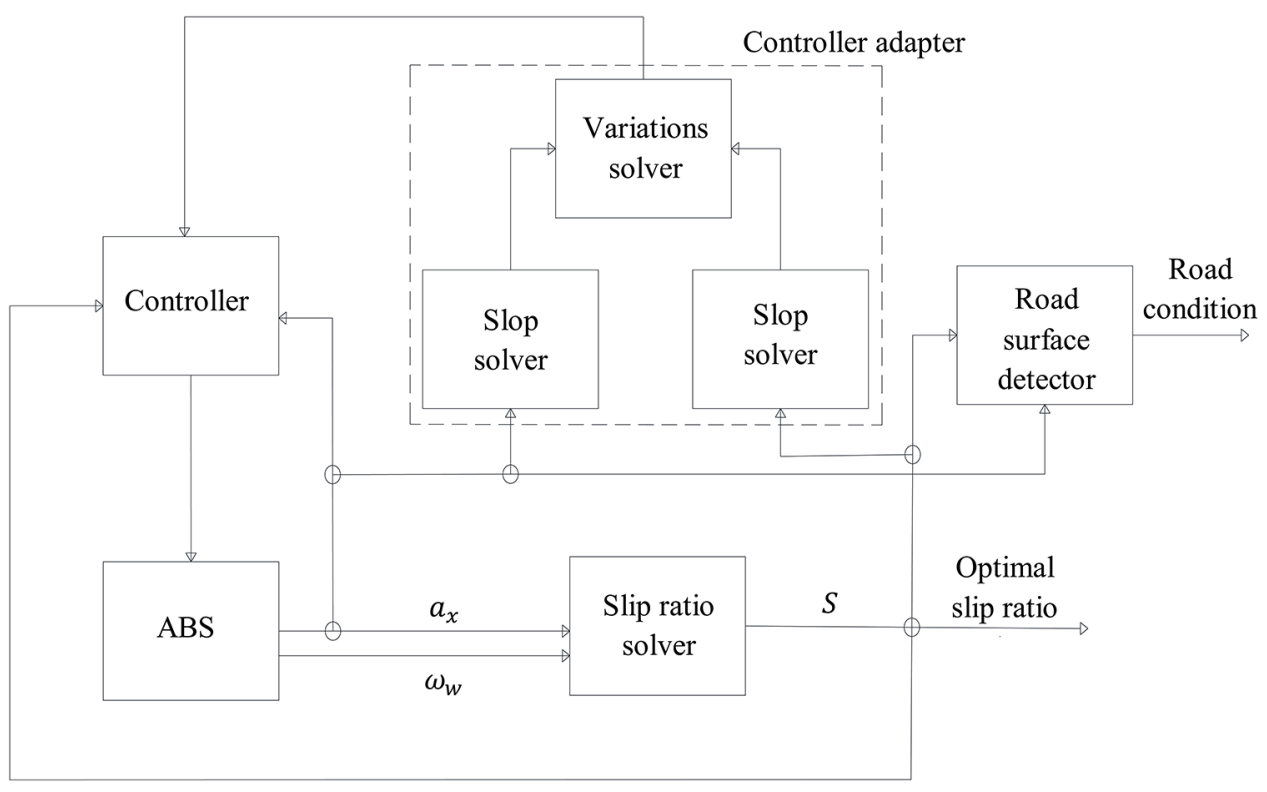

Fig. 4. Block diagram of the control system.

In this study, the self-aligning moment of the tire is considered as $M_{z f}=\left(K_{s} \alpha_{f}\right) N$ that $K_{s}$ is defined as the selfaligning moment stiffness of the tire $[18,42]$.

\section{Anti-lock Brake System (ABS)}

The ABS has some sub-systems such as the vehicle dynamic, the hydraulic system [25] and the tire-road system. The hydraulic system of the ABS consists of the controller and control valves. The control valve input is supplied from the controller. The fluid pressure in the hydraulic system determinates the brake torque and the fluid pressure is regulated by control valves. The regulation of the fluid pressure in the hydraulic system of the ABS must be done to keep the wheel slip ratio in the appropriate range to obtain the lateral stability of the vehicle.

The tire-road system is an important section of the brake system. Variations of the friction between tire and road are resulted from the change of the road surface condition. Furthermore, the controller has a difficult task to keep the vehicle stability. In contact patch area, longitudinal and lateral friction coefficients are available. With increasing the longitudinal friction coefficient during braking, the stop time will be decreased. Meanwhile, with decreasing the lateral friction coefficient, the lateral stability of the vehicle will be decreased. Both of friction coefficients are the functions of the road surface condition and the wheel slip ratio (Fig. 3(a)).

\subsection{Control system strategy}

The operation of the ABS controller is controlling the hydraulic pressure of the brake system to prevent wheel lock-up. Purposes of the ABS are stop time reduction, keeping the lateral stability and preventing the wheel lockup. Mentioned purposes are resulted from increasing the longitudinal and lateral friction coefficients. Both of these friction coefficients depend on the wheel slip ratio and the road surface conditions. According to the variations of friction coefficients with respect to the wheel slip ratio, it is not possible that both of friction coefficients increase simultaneously (see Fig. 3a). On the other hand, the maximum lateral friction coefficient is occurred at zero slip ratio and will be decreased with increasing the slip ratio. However, the zero longitudinal friction coefficient is occurred at zero slip ratio and will be increased with increasing the slip ratio in the stable range of $0 \leq|S| \leq 0.2$. It means that, when the maximum lateral friction coefficient is occurred, the longitudinal friction coefficient goes to zero and the vehicle does not stop. When the maximum longitudinal friction coefficient is occurred, during braking, the maximum force will be occurred in contact patch area and the stop time is decreased. For this manner, the lateral friction coefficient is lower than its maximum but is not enough to causes the lateral instability of the vehicle.

Therefore, when regulation of the wheel slip ratio is occurred in the maximum range of the longitudinal friction coefficient, appropriate results in the lateral friction coefficient will be obtained. According to equation (7), the maximum range of the longitudinal friction coefficient for different conditions of the road surface in this research is happened in the stable range of $0.08-0.18$ for the wheel slip ratio. For the higher amounts of this range, the lower longitudinal friction coefficient will be obtained. It can be concluded that the important strategy of the ABS controller in this study is regulation of the wheel slip ratio in the stable range of $0.08 \leq|S| \leq 0.18$ for different conditions of the road surface (dry, wet and icy).

\subsection{ABS adaptive fuzzy control}

The block diagram for the fuzzy control system of this study is shown in Figure 4. The wheel slip ratio and longitudinal acceleration of the vehicle are considered as 


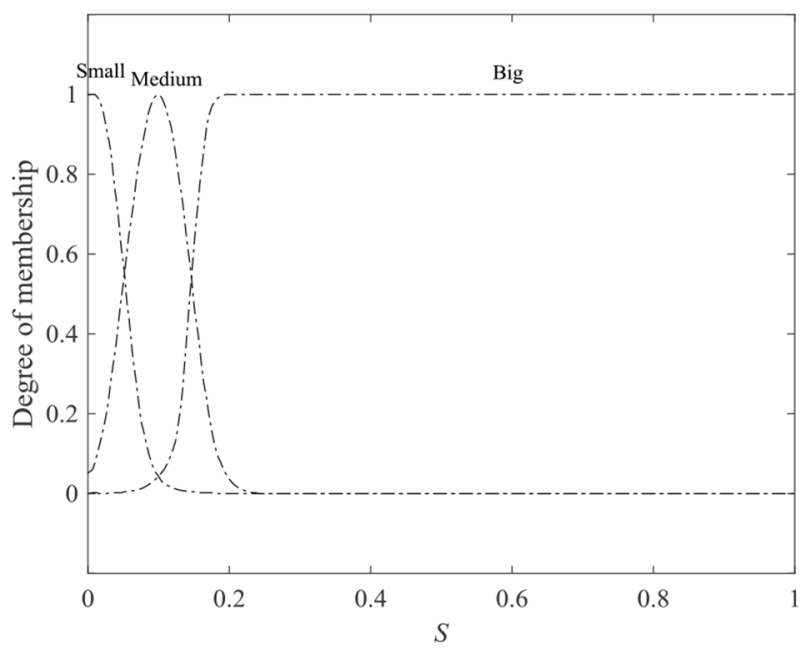

(a)

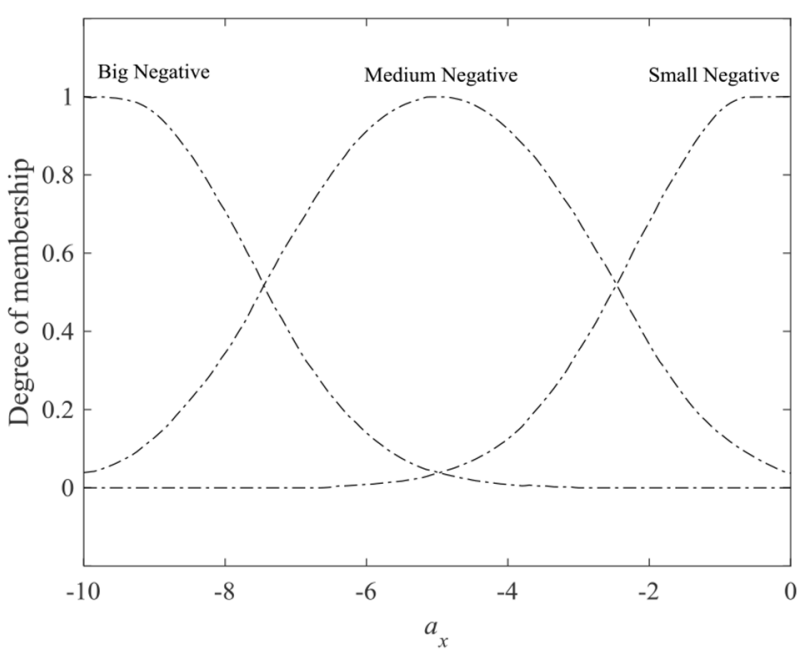

(b)

Fig. 5. Membership functions for the controller inputs: (a) wheel slip ratio and (b) longitudinal acceleration.

two inputs. Mentioned inputs are used in the adaptive fuzzy controller, controller adapter and detector of the road surface condition. Simulation steps of this research are expressed in the following sections.

\subsubsection{Discretization}

The control process of this research is considered in the form of discretization of equations [31-33]. As an example, discrete equations of the wheel rotation and longitudinal force of the tire are considered as:

$$
\begin{gathered}
\omega_{w}(i+1)=\omega_{w}(i)+\left(\frac{\tau}{I_{w}}\right)\left(-T_{b}(i)-r_{w} F_{x f}(i)\right) \\
F_{x f}(i)=-\mu(S(i+1)) . m g
\end{gathered}
$$

That $\tau$ is the sampling time and $i$ shows each sampling period of the process.

\subsubsection{Adaptive fuzzy controller}

The wheel slip ratio and longitudinal acceleration of the vehicle are considered for regulation of control rules. Gaussian membership functions for the controller inputs are shown in Figure 5. Fuzzy IF-THEN rules such as "IF THE SLIP RATIO IS MEDIUM AND; THE NEGATIVE LONGITUDINAL ACCELERATION IS BIG; THEN THE CONTROL SIGNAL IS BIG" and "IF THE SLIP RATIO IS SMALL; THEN THE CONTROL SIGNAL IS VERY BIG" are considered for the control process. These rules are evaluated according to the slip ratio and the longitudinal acceleration. The inference process is performed by the Mamdani max-min inference method $[35,36]$. To obtain the control law ( $u$ is resulted from the controller output) the defuzzifier process is done for inference of results [37]. In this study, the hydraulic system of the ABS is considered as an actuator with first-order dynamic [41].
The discrete dynamic of the actuator is considered as equation (13).

$$
T_{b}(i+1)=T_{b}(i)+20.37 \tau\left(u(i)-T_{b}(i)\right)
$$

The adaptive fuzzy controller consists of the linguistic base, the inference engine and the defuzzifier section. The adaptive fuzzy controller of this study is created in form of a matrix by a function in the MATLAB software. When elements of the controller matrix are constant, the control system does not have a good performance due to change of the road surface condition. However, when elements of the controller matrix have the adaptive property then the adaptive property of the controller is appeared. Due to the adaptive property such as the online parameter estimation, the wheel slip ratio will be regulated in the optimal value for different conditions of the road surface. The procedure of using the controller matrix is shown in the equation (14).

$$
u(i+1)=\operatorname{evalfis}\left[S(i+1) \quad a_{x}(i+1)\right]
$$

According to equation (14) the controller matrix is created owning to the new adaptive parameter and it is obtained by the controller adapter. Therefore, the control law of each period $(u(i+1))$ is obtained due to two inputs of the wheel slip ratio and longitudinal acceleration of each sampling period $\left(S(i+1)\right.$ and $\left.a_{x}(i+1)\right)$.

\subsubsection{Controller adapter}

The controller adapter in this study consists of solver of variations slop and solver of parameters' variations. In the proposed controller adapter, parameters of the fuzzy controller are regulated to obtain the appropriate slip ratio by the control law. The solver of variations slop performs the derivation and the relation between variations of the wheel slip ratio and longitudinal acceleration, forms the adaptive basis of the controller. 


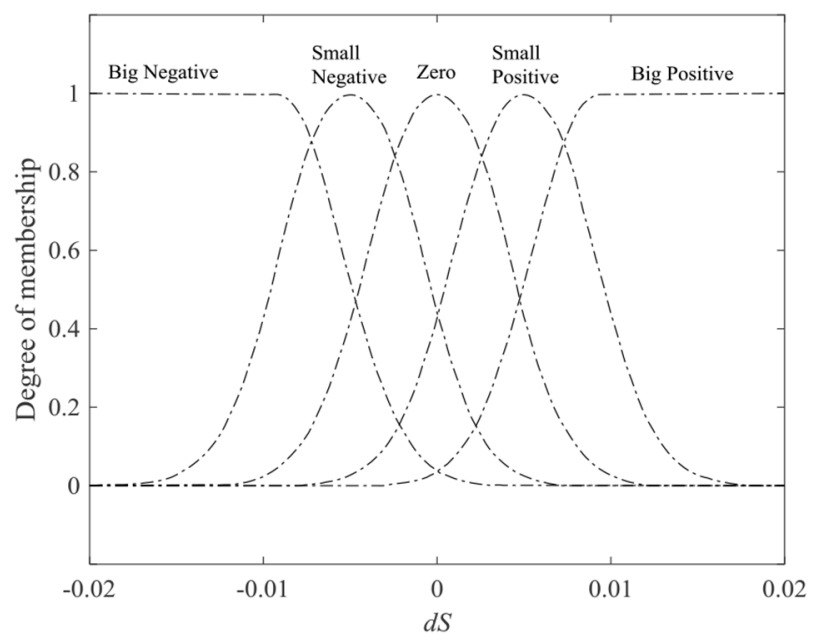

(a)

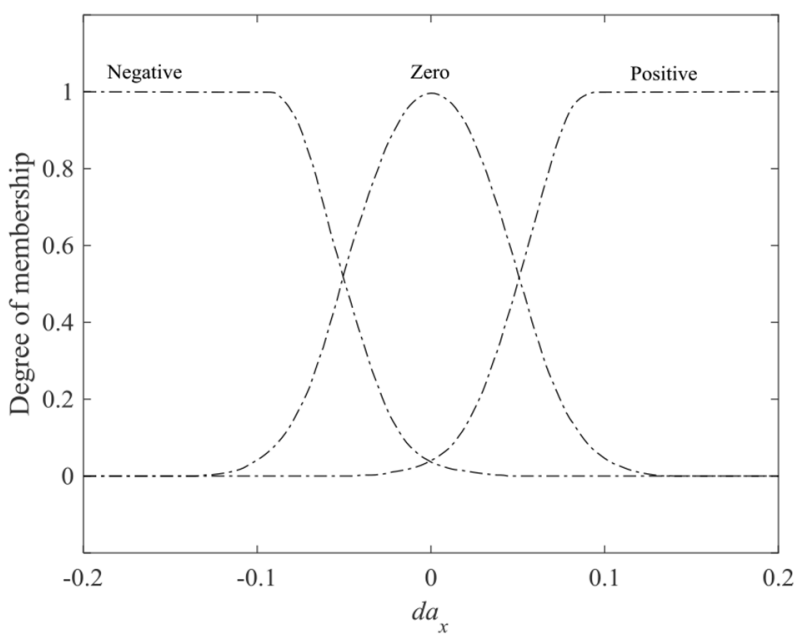

(b)

Fig. 6. Membership functions for solver inputs of the parameters' variations: (a) variations of the wheel slip ratio and (b) variations of the longitudinal acceleration.

The appropriate variations of controller parameters for regulating the wheel slip ratio in the optimal value are resulted from the solver of parameters' variations. The solver of parameters' variations consists of the knowledge base, the inference engine and the defuzzifier section as like as the fuzzy controller. For the knowledge base Gaussian membership functions of inputs are considered as Figure 6 . In the knowledge base, fuzzy IF-THEN rules such as "IF VARIATIONS OF THE SLIP RATIO IS ZERO AND; VARIATIONS OF THE LONGITUDINAL ACCELERATION IS ZERO; THEN INCREASE OF PARAMETERS IS ZERO" and "IF VARIATIONS OF THE SLIP RATIO IS BIG NEGATIVE AND; VARIATIONS OF THE LONGITUDINAL ACCELERATION IS SMALL POSITIVE; THEN INCREASE OF PARAMETERS IS BIG" are used to present appropriate output. These rules are inferenced by the Mamdani max-min inference method.

The defuzzifier process is performed by the centroid of area method for the final result and the final result will be sent to the controller. The control adapter of this study is created in the form of a matrix by a function in the MATLAB software. Equation (15) shows the instruction of the control adapter matrix.

$$
\begin{aligned}
& \text { Adaptive parameter }(i+1)=\text { evalfis }[\Delta(d S(i+1) / d t) \\
& \left.\quad \Delta\left(d a_{x}(i+1) / d t\right)\right]
\end{aligned}
$$

Equation (15) illustrates that with using the matrix of the controller adapter and according to variations of the wheel slip ratio slop and variations of the longitudinal acceleration slop the parameter which adaptive process is performed by it, will be obtained.

\subsubsection{Detector of the road surface condition}

The detector of the road surface condition is used for regulation of fuzzy controller rules. For each road surface condition, a special number could be considered. For instance, number one introduces the surface of dry road and the number zero shows the surface of icy road and other conditions of the road surface such as surface of wet road places between this range $([0,1])$. The detector of the road surface condition consists of the knowledge base, the inference engine and the defuzzifier section. Similar to the controller, Gaussian membership functions for detector inputs of the road surface condition are considered as Figure 7. Fuzzy IF-THEN rules such as "IF THE LONGITUDINAL ACCELERATION IS BIG NEGATIVE; THEN THE ROAD SURFACE CONDITION IS DRY" and "IF THE SLIP RATIO IS MEDIUM AND; THE LONGITUDINAL ACCELERATION IS MEDIUM NEGATIVE; THEN THE ROAD SURFACE CONDITION IS WET" are used to detective of the road surface condition. These rules are evaluated by the Mamdani max-min inference method and after the defuzzifier process, the final result will be obtained and will be placed in the range of $[0,1]$. Detector matrix of the road surface condition is created by a function in the MATLAB software and its instruction is presented in equation (16).

$$
\begin{aligned}
& \text { Detect of the road surface condition }(i+1) \\
& \quad=\text { evalfis }\left[S(i+1) \quad a_{x}(i+1)\right]
\end{aligned}
$$

According to equation (16), with using the wheel slip ratio and the longitudinal acceleration of $i$ period and detector matrix of the road surface condition, the road surface condition of each sampling period $(i)$ will be detected in form of a number in the range of $[0,1]$.

\section{Simulation results}

To evaluate the performance of the anti-lock brake system and the vehicle stability during braking and steering input, the vehicle is considered as the class of SUVs (Jack S5) with parameters of Table 1. For different conditions of the road surface the steering angle of this research is considered as Figure 8. The ratio of the maximum steering angle of 


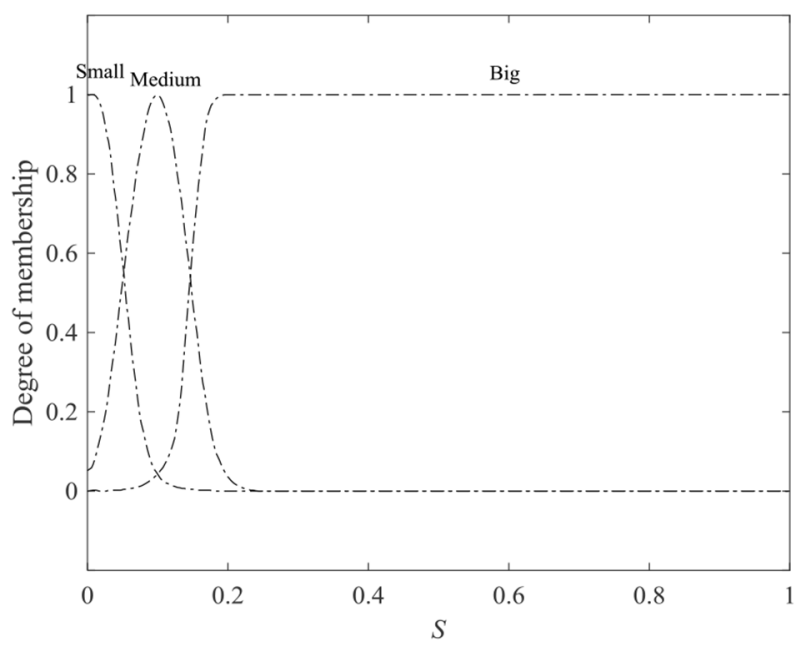

(a)

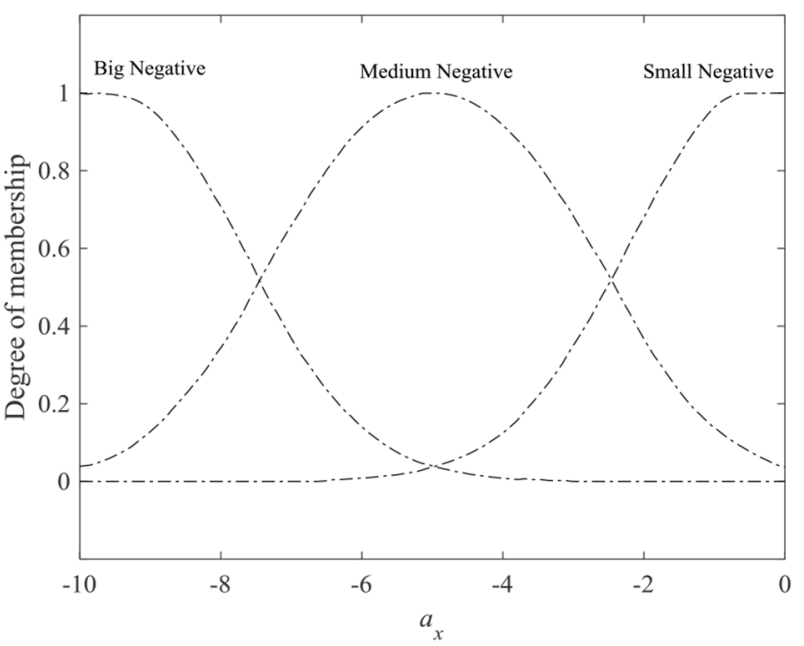

(b)

Fig. 7. Membership functions for detector inputs of the road surface condition: (a) wheel slip ratio and (b) longitudinal acceleration.

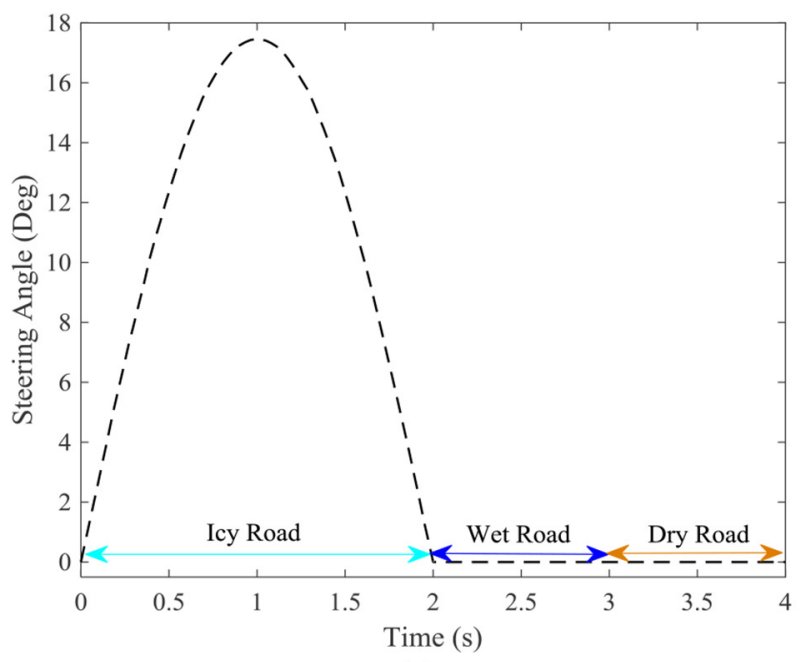

(a)

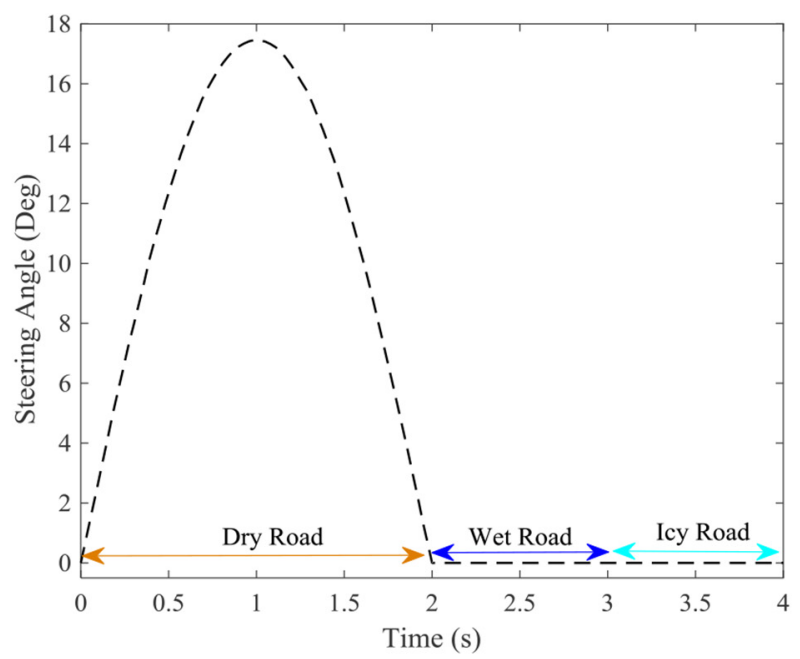

(b)

Fig. 8. Steering angle for: (a) icy, wet and dry road surfaces and (b) dry, wet and icy road surfaces.

Table 1. System parameters of the case study.

\begin{tabular}{lll}
\hline Parameter & Value & Unit \\
\hline$m$ & 1545 & $\mathrm{~kg}$ \\
$I_{z z}$ & 1808 & $\mathrm{Kg} \cdot \mathrm{m}^{2}$ \\
$K_{s}$ & 3500 & $\mathrm{~N} \cdot \mathrm{m} / \mathrm{rad}$ \\
$K_{c}$ & 120 & $\mathrm{kN} / \mathrm{rad}$ \\
$\tau$ & 5 & $\mathrm{~ms}$ \\
$I_{w}$ & 3.04 & $\mathrm{Kg} \cdot \mathrm{m}^{2}$ \\
$d$ & 1.84 & $\mathrm{~m}$ \\
$V_{0 x}$ & 60 & $\mathrm{Km} / \mathrm{h}$ \\
$l_{f}$ & 1.103 & $\mathrm{~m}$ \\
Tire type & & $225 / 55 \mathrm{R} 18$ \\
Numerical model of tire & Pacejka Magic Formula \\
\hline
\end{tabular}

Figure 8 to the maximum angle of the front wheel is 17.5 to 1 . On the other hand, according to Figure 8 the maximum variation in the angle of front wheel of is one degree.

In Figure 8a for different times $(0 \leq t \leq 4)$ the road surface condition is changed from icy to wet and dry. Figure $8 \mathrm{~b}$ also shows that for $0 \leq t \leq 4$ the road surface condition is changed from dry to wet and icy. Variations of the longitudinal friction coefficient for dry, wet and icy road surfaces are shown in Figure 9. Figure 9 shows that the maximum of the longitudinal friction coefficient with respect to the wheel slip ratio $(\mu(S)$ curves) for dry, wet and icy surfaces of the road is: $1(0.18), 0.7(0.13)$ and $0.3(0.08)$; respectively. For the road surface conditions of Figure 8a, variations of the vehicle longitudinal velocity and angular velocity of the wheel is shown in Figure 10. Figure 11 shows tracking the optimal wheel slip ratio for different conditions of the road surface. 


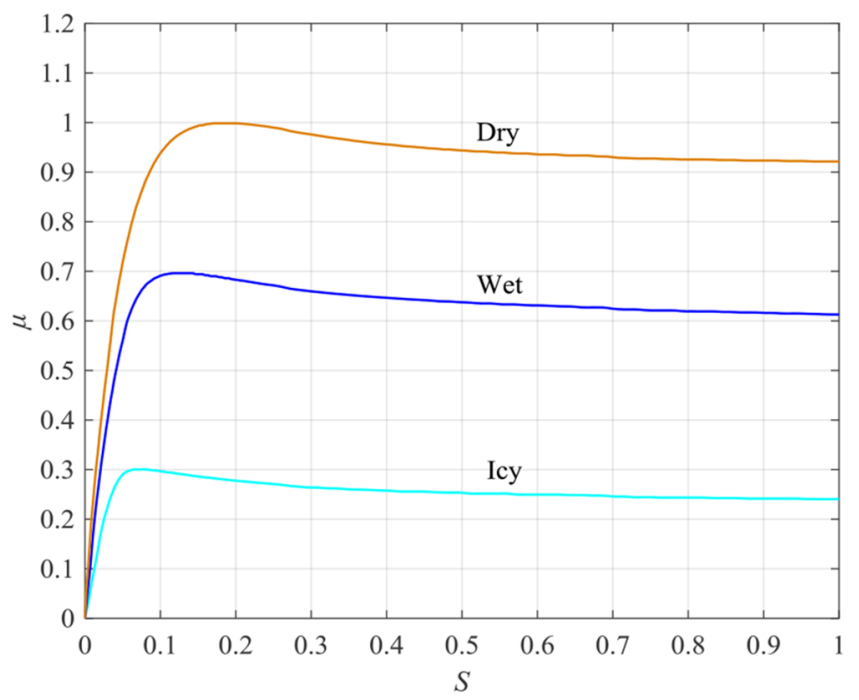

Fig. 9. $\mu(S)$ curves for different conditions of the road surface.

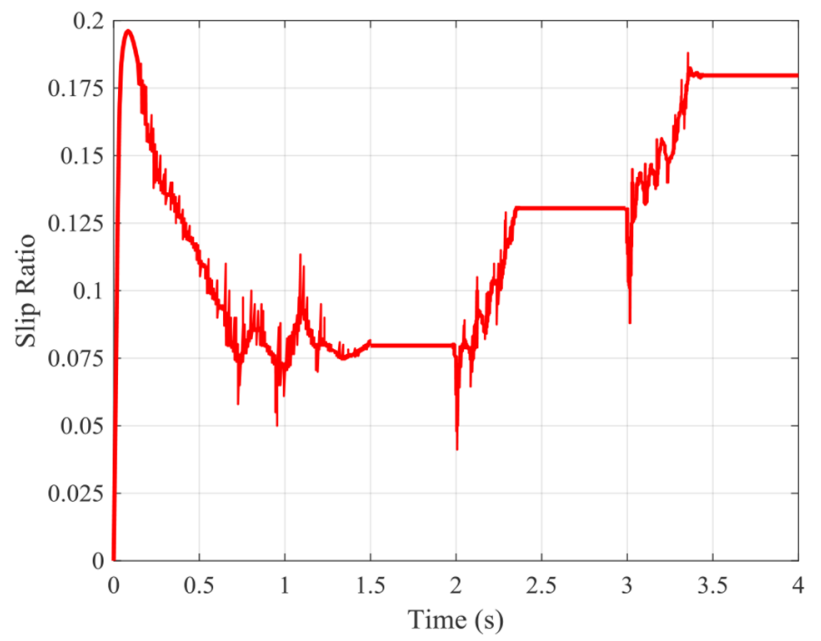

(a)

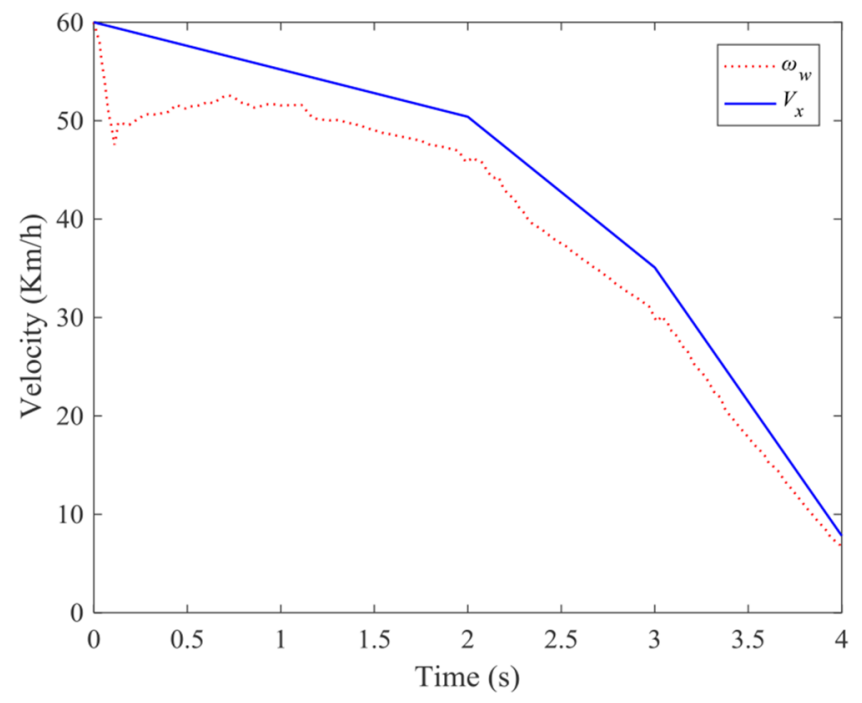

Fig. 10. Variations of the vehicle longitudinal velocity and angular velocity of the wheel for road surfaces of Figure 8a.

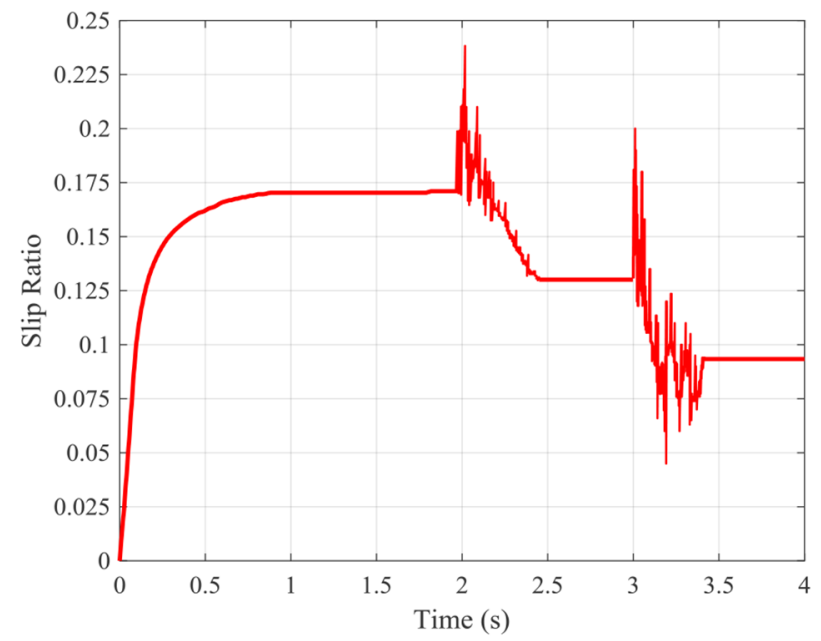

(b)

Fig. 11. Tracking the optimal slip ratio for road surfaces of: (a) Figure. 8a and (b) Figure 8b.

During braking for different conditions of the road surface, according to results of the vehicle longitudinal velocity and angular velocity of the wheel (Fig. 10) it can be concluded that the stop time of the vehicle will be decreased. According to Figure 11, tracking the wheel slip ratio is ascertained by the controller with a high accuracy for different conditions of the road surface. On the other hand, the proposed intelligent controller regulates the wheel slip ratio in the optimal ranges with changing the road surface condition from dry to wet and icy and vice versa. In regard to the change in the condition of the road surface, the control system meets a problem. The proposed control system works well against this problem and tracks the optimal wheel slip ratio. According to the results it can be concluded that the proposed adaptive fuzzy controller could be effective to obtain purposes of the anti-lock brake system.
During braking and steering input on a slippery road (Fig. 8 a for $0 \leq t \leq 2$ ), the dynamic behavior of the vehicle such as lateral velocity and rate of yaw angle with parameters of Table 1 is shown in Figures 12 and 13 by dot lines. The effective parameters of the wheel such as tire stiffnesses (the self-aligning moment stiffness and sideslip force stiffness of the tire) and the steering input are selected for this research. The influence of the effective parameters on the dynamic behavior of the vehicle is shown in Figures 12 and 13 by dash and dash-dot lines.

Figure 12 shows that with increasing tire stiffnesses such as the self-aligning moment stiffness and sideslip force stiffness of the tire, system variables are rapidly converged to zero. On the other hand, the resistance of system variables increases to lateral stimulation and the vehicle stability will be increased. Practically, radial tires with low profile and wider width are used for this purpose. Figure 13 

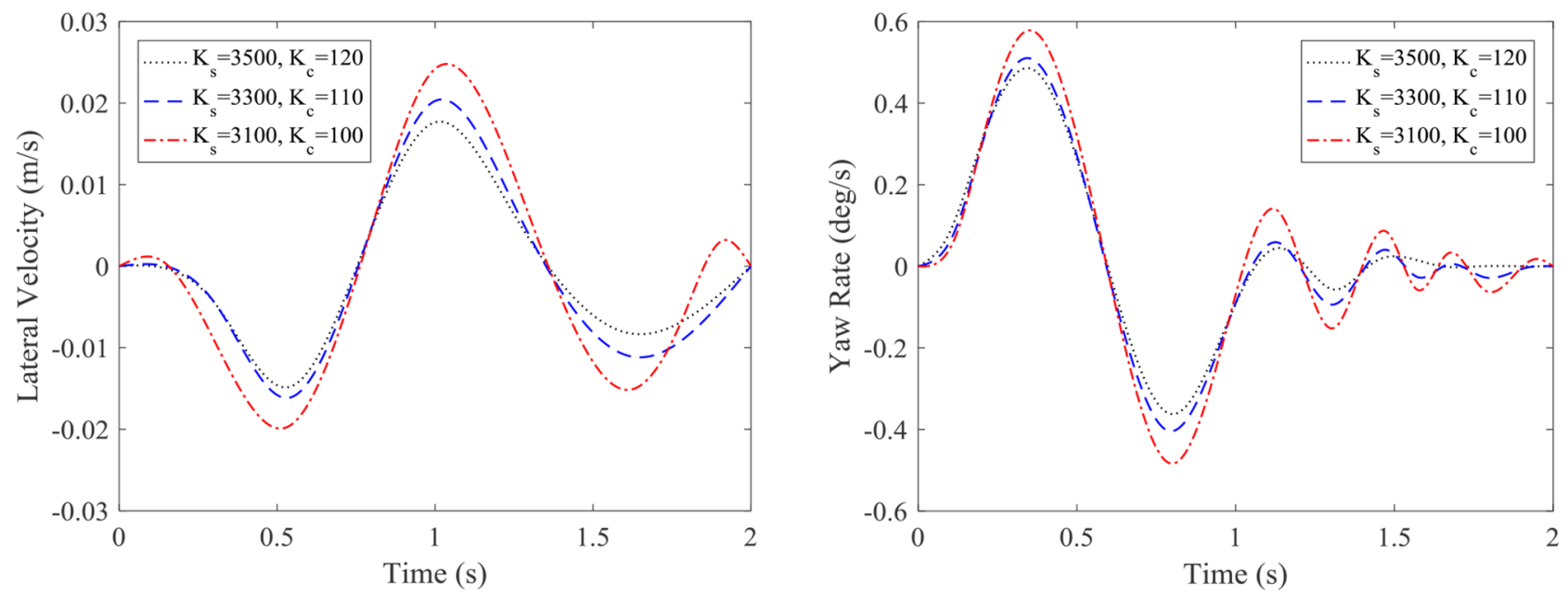

Fig. 12. Influence of the self-aligning moment stiffness and sideslip force stiffness of the tire on the vehicle's dynamic behavior during braking and steering input.
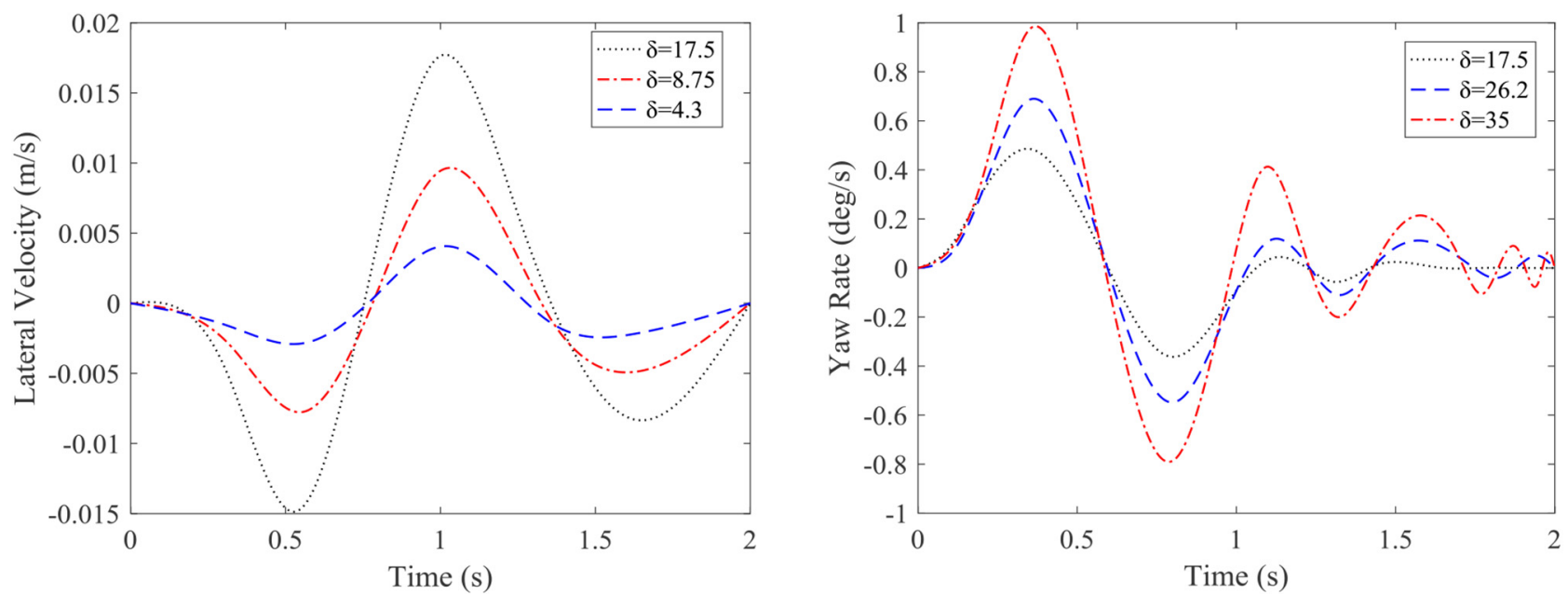

Fig. 13. Influence of the steering input on the vehicle's dynamic behavior during braking.

shows that by raising the steering input, system variables are slowly converged to zero. It should be noted that in general, the parameters that cause change in the load transfer during braking (the height of the center of gravity and tire stiffnesses), will have a great effect on the convergence and resistance of system variables.

\section{Summary and conclusion}

In this research, the stability of the class of SUVs (Jack S5) during braking and steering input is investigated. The vehicle model is considered as a quarter-car model with the anti-lock brake system (ABS). The Pacejka non-linear Magic Formula is used for the investigation of tire behavior and nonlinear equations of the dynamic model are selected. For the quarter-car model of this study, wheel slip ratio regulation in the maximum range of longitudinal friction coefficient for different conditions of the road surface is selected as the purpose of the ABS control. The adaptive fuzzy controller is considered and regulated in this study according to variations of longitudinal acceleration of the vehicle and the wheel slip ratio. In the adaptive fuzzy control of this study because of the online parameter estimation, controller parameters are regulated to control the fluid pressure of the brake hydraulic system. During braking and steering input on a slippery road, the influence of the wheel parameters is investigated on the dynamic behavior and the system stability. Based on the results, the maximum longitudinal friction coefficient with respect to the wheel slip ratio $(\mu(S))$ for dry, wet and icy surfaces of the road is obtained as $1(0.18), 0.7(0.13)$ and $0.3(0.08)$. For different conditions of the road surface tracking the optimal wheel slip ratio with a high accuracy is performed by the proposed controller. For different conditions of the road surface according to the results of longitudinal velocity of the vehicle and angular velocity of the wheel, the stop time of the vehicle during braking will be decreased. During braking and steering input on a slippery road, when tire stiffnesses are increased the resistance of 
system variables increases to lateral stimulation and the vehicle stability will be increased. This result also satisfies for reduction of the steering input.

\section{References}

[1] M.C. Walz, Trends in the static stability factor of passenger cars, light trucks, and vans, (2005)

[2] F. Farroni, M. Russo, R. Russo, M. Terzo, F. Timpone, A combined use of phase plane and handling diagram method to study the influence of tyre and vehicle characteristics on stability, Vehicle System Dynamics 51, 1265-1285 (2013)

[3] Y. Hisaoka, M. Yamamoto, A. Okada, Closed-loop analysis of vehicle behavior during braking in a turn, JSAE review $\mathbf{2 0}$, 537-542 (1999)

[4] J. Lenasi, G. Danon, S. Zežclj, Lateral stability of a braking vehicle on the friction limit, Vehicle System Dynamics 29, 711-716 (1998)

[5] Q. Qu, Y. Liu, On lateral dynamics of vehicles based on nonlinear characteristics of tires, Vehicle system dynamics 34, 131-141 (2000)

[6] B. Olson, S. Shaw, G. Stépán, Stability and bifurcation of longitudinal vehicle braking, Nonlinear Dynamics 40, 339-365 (2005)

[7] M. Ahmadian, Integrating electromechanical systems in commercial vehicles for improved handling, stability, and comfort, SAE International Journal of Commercial Vehicles 7, 535-587 (2014)

[8] A.H. Kazemian, M. Fooladi, H. Darijani, Rollover index for the diagnosis of tripped and untripped rollovers, Latin American Journal of Solids and Structures 14, 1979-1999 (2017)

[9] A.H. Kazemian, M. Fooladi, H. Darijani, Non-linear control of vehicle's rollover using sliding mode controller for new 8 degrees of freedom suspension model, International Journal of Heavy Vehicle Systems 26, 707-726 (2019)

[10] E. Joa, K. Yi, Y. Hyun, Estimation of the tire slip angle under various road conditions without tire-road information for vehicle stability control, Control Engineering Practice 86, 129-143 (2019)

[11] S. Cheng, L. Li, B. Yan, C. Liu, X. Wang, J. Fang, Simultaneous estimation of tire side-slip angle and lateral tire force for vehicle lateral stability control, Mechanical Systems and Signal Processing 132, 168-182 (2019)

[12] M. Reiter, J. Wagner, Automated automotive tire inflation system-effect of tire pressure on vehicle handling, IFAC Proceedings Volumes 43, 638-643 (2010)

[13] Y. Zhang, A. Khajepour, E. Hashemi, Y. Qin, Y. Huang, Reconfigurable model predictive control for articulated vehicle stability with experimental validation, IEEE Transactions on Transportation Electrification 6, 308-317 (2020)

[14] V. Rezaei, A.M. Shafei, Dynamic analysis of flexible robotic manipulators constructed of functionally graded materials, Iranian Journal of Science and Technology, Transactions of Mechanical Engineering 43, 327-342 (2019)

[15] A. Janot, P.M. Wensing, Sequential semidefinite optimization for physically and statistically consistent robot identification, Control Engineering Practice 107, 104699 (2021)
[16] Y. Zhang, H. Liu, T. Ma, L. Hao, Z. Li, A comprehensive dynamic model for pneumatic artificial muscles considering different input frequencies and mechanical loads, Mechanical Systems and Signal Processing 148, 107133 (2021)

[17] C.-Y. Lu, M.-C. Shih, Application of the pacejka magic formula tyre model on a study of a hydraulic anti-lock braking system for a light motorcycle, Vehicle System Dynamics 41, 431-448 (2004)

[18] T.D. Gillespie, Fundamentals of vehicle dynamics, Society of automotive engineers Warrendale, PA, 1992

[19] H. Pacejka, Tire and vehicle dynamics, Elsevier, 2005

[20] E. Bakker, H.B. Pacejka, L. Lidner, A new tire model with an application in vehicle dynamics studies, SAE Transactions 98, 101-113 (1989)

[21] Y. Lee, S.H. Zak, Designing a genetic neural fuzzy antilockbrake-system controller, IEEE Transactions on Evolutionary Computation 6, 198-211 (2002)

[22] J.R. Layne, K.M. Passino, S. Yurkovich, Fuzzy learning control for antiskid braking systems, IEEE Transactions on Control Systems Technology 1, 122-129 (1993)

[23] D. Madau, F. Yuan, L. Davis, L. Feldkamp, Fuzzy logic antilock brake system for a limited range coefficient of friction surface, [Proceedings 1993] Second IEEE International Conference on Fuzzy Systems, IEEE, 1993, pp. 883-888

[24] S. Germann, M. Wurtenberger, A. Daiss, Monitoring of the friction coefficient between tyre and road surface, Proceedings of the third IEEE Conference on Control Applications, 1994, pp. 613-618

[25] A. Mirzaei, M. Moallem, B. Mirzaeian, B. Fahimi, Design of an optimal fuzzy controller for antilock braking systems, 2005 IEEE Vehicle Power and Propulsion Conference, IEEE, 2005, pp. 823-828

[26] H. Mirzaeinejad, M. Mirzaei, A new approach for modelling and control of two-wheel anti-lock brake systems, Proceedings of the Institution of Mechanical Engineers, Part K: Journal of Multi-body Dynamics 225, 179-192 (2011)

[27] E. Šabanovič, V. Zuraulis, O. Prentkovskis, V. Skrickij, Identification of Road-Surface Type Using Deep Neural Networks for Friction Coefficient Estimation, Sensors 20, $612(2020)$

[28] A. Aksjonov, V. Ricciardi, K. Augsburg, V. Vodovozov, E. Petlenkov, Hardware-in-the-loop test of an open loop fuzzy control method for decoupled electro-hydraulic antilock braking System, IEEE Transactions on Fuzzy Systems (2020)

[29] J. Zhang, S. Zhou, J. Zhao, Nonlinear robust wheel slip rate tracking control for autonomous vehicle with actuator dynamics, Advances in Mechanical Engineering 12, $1687814020925222(2020)$

[30] P. Wellstead, N. Pettit, Analysis and redesign of an antilock brake system controller, IEE Proceedings-Control Theory and Applications 144, 413-426 (1997)

[31] S. Jagannathan, M. Vandegrift, F.L. Lewis, Adaptive fuzzy logic control of discrete-time dynamical systems, Automatica 36, 229-241 (2000)

[32] H. Li, Y. Wu, M. Chen, Adaptive fault-tolerant tracking control for discrete-time multiagent systems via reinforcement learning algorithm, IEEE Transactions on Cybernetics 51, 1163-1174 (2020)

[33] K. Sakai, H. Shibasaki, R. Tanaka, T. Murakami, Y. Ishida, A design of a robust discrete-time controller, ISA Transactions 56, 155-164 (2015) 
[34] S. Zheng, H. Tang, Z. Han, Y. Zhang, Controller design for vehicle stability enhancement, Control Engineering Practice 14, 1413-1421 (2006)

[35] E. Mamdani, Applications of fuzzy set theory to control systems: a survey, Fuzzy Automata and Decision Processes, 77-88 (1977)

[36] B.P. Graham, R.B. Newell, Fuzzy adaptive control of a first-order process, Fuzzy Sets and Systems 31, 47-65 (1989)

[37] Z. Liu, S. Lu, R.-h. Du, A genetic-fuzzy control method for regenerative braking in electric vehicle, International Journal of Computing Science and Mathematics 11, 263-277 (2020)

[38] G.F. Mauer, A fuzzy logic controller for an ABS braking system, IEEE Transactions on Fuzzy Systems 3, 381-388 (1995)
[39] S. Latreche, S. Benaggoune, Robust wheel slip for vehicle anti-lock braking system with Fuzzy Sliding Mode Controller (FSMC), Engineering, Technology \& Applied Science Research 10, 6368-6373 (2020)

[40] W. Li, H. Du, W. Li, A modified extreme seeking-based adaptive fuzzy sliding mode control scheme for vehicle antilock braking, International Journal of Vehicle Autonomous Systems 15, 1-25 (2020)

[41] C. Acosta-Lú, S.D. Gennaro, M.E. Sánchez-Morales, An adaptive controller applied to an anti-lock braking system laboratory, Dyna 83, 69-77 (2016)

[42] M. Massaro, V. Cossalter, G. Cusimano, The effect of the inflation pressure on the tyre properties and the motorcycle stability, Proceedings of the Institution of Mechanical Engineers, Part D: Journal of Automobile Engineering 227, 1480-1488 (2013)

Cite this article as: A. Shahabi, A.H. Kazemian, S. Farahat, F. Sarhaddi, Wheel slip ratio regulation for investigating the vehicle's dynamic behavior during braking and steering input, Mechanics \& Industry 22, 17 (2021) 